\title{
Smart Parking System Using Color QR Code
}

\section{Sarat Kumar sahoo a, , Rashmita khilar b, Samiksha Nayak c, Mary Rexcy Asha d, Ann Jerin Amalorpavaraj d}

a Professor, Department of Electrical Engineering, Parala Maharaja Engineering College Berhampur, Odisha, India

${ }^{b}$ Assistant Professor, Panimalar Engineering College, Chennai, Tamil Nadu, India

c School of Electrical Engineering, VIT University, Vellore, Tamil Nadu, India

d Associate Professor, Panimalar Engineering College, Chennai, Tamil Nadu, India

e Sri Shakthi Institute of Engineering Technology, Coimbatore, Tamil Nadu, India

${ }^{*}$ Corresponding Author sarata1@rediffmail.com (Sarat Kumar sahoo)

Received : 07 04 2019 Accepted : 16 05 2019

\begin{abstract}
In today's world, parking area constitutes nearly most of traffic congestion is caused by vehicles cruising around their destination and looking for a place to park. Due to this reason many day to day activities are affected such as waste of time, fuel wastage, frustration to drivers, theft fear, pollution etc. These factors motivated to pave a new method for smart parking system. In this method the detection is reliable, even when tests are performed using images captured from a different viewpoint. It also provides to design a highly reliable \& compatible image segmentation measures for parking slot identification system and a user key driven data base measures to detect the vehicle using theft alarm system.
\end{abstract}

Keywords:Smart parking, Image processing, Vehicle, Classifier.

\section{Introduction}

In this developing world, there are many parking areas. The ground sensors are used to determine the status of the various parking spaces. The above scenario requires the installation and maintenance of sensors in every parking places and in turn parking slots. Implementing sensors in every parking place is highly expensive, especially in wide area parking facility. There is an increase in number of vehicles used over the years it causes significant parking issues. There are various technologies and techniques for identifying the car parking space detection which involves effective management of parking lots without any tension. To overcome the above disadvantages such as waste of time, fuel wastage, frustration to drivers, theft fear, pollution and limited space availability etc [1]. The above factors motivated the researchers to develop a new method for smart parking system. In today's scenario nearly one fifth of the human population travel in their own vehicles, Rather than public transport. In the near future this rate will increase to a bigger value. Hence the rate of people using private vehicles becomes more with the rise in human population. Nowadays, the construction of multiplexes, theatres, and shopping malls becomes high and every place requires easy parking system. To solve this problem, the search for an efficient parking system is required which could effectively manage in parking of vehicles. By knowing the real time availability of free parking spaces and communicating to the users can be of great help in reducing the queues, improve scalability, and the time required to find an empty space in a parking lot. Therefore, this paper propose a system which is highly desirable to have a quick and cost effective way to track and guide drivers to available parking spaces. The proposed system uses cameras to monitor the vehicles that will provide efficient result for identifying parking spaces by using artificial neural network classifier and it is cost efficient. It doesn't require more investment, maintenance and provide more security using QR code generated for the user for a parking lot that will be given to every user who wants to protect their vehicles that enters into the premise. For leaving the premise one should report the parking lot that is already provided. This will ensure high security to the vehicle and manages the parking area efficiently.

The smart parking system will provide an efficient solution to the parking difficulties faced by the people by proving a parking lot and providing a parking lot to park their vehicles in a secured way. By this the security becomes unquestionable and it will reduce manpower [2].

\section{Related Work}

The use of image based approaches for vacant parking space detection has attracted considerable interest from the research community over the years to replace with sensor based techniques. A review on smart car parking systems is summarized as follows. [1] Proposed a system in 
which the system can distinguish the parking slot availability of moving or small objects, cost effective reliable, occupancy classification stage of the recognized parking slots solves parking slot availability classification issues in the year 2016. The algorithm for vacant parking space includes a slot validation step that identifies multiple slots in a probabilistic integration approach and has more flexibility on irregular patterns slot occupancy in the classification stage, reliable parking availability detection is achieved through visual slot features, including the Histogram of Gradient and frequency magnitude features, via a Support Vector Machine. But it had some drawbacks like temporal information is not included and there is room for the improvement of slot recognition.

Giuseppe Amato, Fabio Carrara, FabrizioFalchi, Claudio Gennaro and Claudio Vairo [2] suggested an approach for the detection of car parking occupancy based on Deep Learning in 2016.The main advantage of this method is high accuracy and many benefits are present in the raspberry pie camera. It uses Convolutional Neural Networks to classify the parking space occupancy directly on board of a Raspberry Pi camera. But, it provides some inconveniences like the presence of noise due to light conditions variation, shadows. Although it gives many advantages in raspberry pi camera, it also has some disadvantages like an in depth analysis of the performance in non ideal situations such as night, foggy, or snowy Condition.

In the year 2013, [3] proposed a system which uses the method of Edge Detection, Boundary extraction. The above method provides some advantages like cost effective for robust image based. Vacant space detection system could be deployed in both indoors and outdoors. In order to achieve robustness, the information related to parking information is shown on display boards at the entrance of the parking building. But this method of edge detection, boundary, features foreground and background subtraction method also lead to the disadvantages like inefficient when the color of the vehicle is very similar to the background, and the method did not provide parking vacancy information to drivers through mobile phones. The classifiers are not used for classifying the images. The security issue is not considered.

Another approach was suggested by [4] 2009 proposed a paper using OBU On Board Units, RSU Road Side Unit, RSU's guard to detect the anomaly many RSU's, Trust Authority is used which thereby produced advantages like easy at peak hours ,no frustration ,no sensors, no theft. Some disadvantages are that all vehicles must have OBU, no mechanism is implemented to track the stolen vehicle, which is driven away, over trust over Trust Authority Even if the vehicle is different from its position, it gives a warning, and detection period is long.

[5] this paper gives some advantages like cheap and easy installed because of the simple equipment. This method was invented in 2011 that drivers can get useful and real time information related to the parking lot through the information display. It uses the steps like Image acquisition, RGB to gray conversion, Image enhancement, Image matching using edge detection. The disadvantages are Time consumption required for processing the image eventually the accuracy is less.

[6] suggested the usage of matlab for the image processing for parking slot detection .Some of its advantages are the camera can be used to sense the empty space through video image detection, noise can be removed with the help of a technique called Morphology, Exact Edge and outline Boundaries of an Image is obtained in image processing for parking slot detection. The drawbacks of the above system is when the object is moving at high speed, it becomes tedious to take snap on that image, as the whole Sensors can be also a problem.

[7] suggested a Vision based Vacant Parking Lot Detection which had the facilities to overcome the problems of changing lighting and shadow effects.For better performance of the above approach the researchers have introduce a new transitional state called "Transition" which represents the passing of a vacant parking space. The method suggested here is combination of the Adaptive Background Subtraction, algorithm and Speeded up Robust, Features algorithm. Although it produces some disadvantages like suffering in case of unexpected scenario like the presence of pedestrians or the inter objects occlusion.

[8] derived an Intelligent Parking Management System Based on Image Processing. This method contains some advantages that overcomes the defects by the automatic parking system is used to make the whole process of parking cars more efficient and less complex for both drivers and administrators. They proposed the method of Intelligent Parking Management System.But it produces some defects like current limitation in this paper is the weather conditions.

In Identifying Parking Spaces \& Detecting Occupancy suggested by [9] provides the advantages like city wide parking management system can be quickly deployed at low cost by using machine learning technique named as vision based IOT that dynamically identify the parking spaces without any manual specification. Its disadvantages can be grouped as camera based parking space occupancy a system at night time is challenging. 


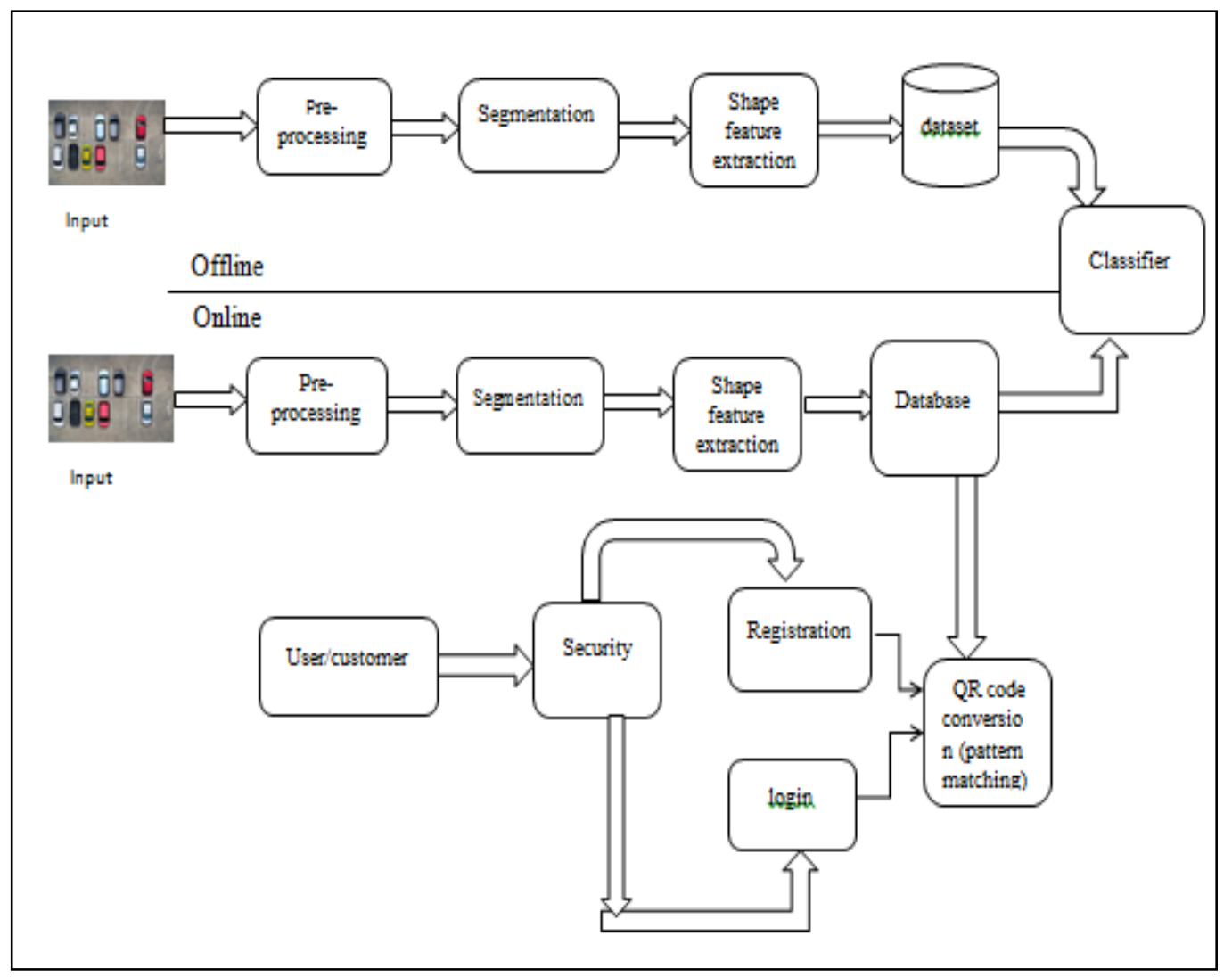

Fig1. System architecture

\section{Methodology}

\section{A. System Architecture}

Figure: 1shows the parking slot identification with deployed security for the detected vehicle in a step by step process flow chart and the first step of the process begins with the Image processing. It denotes a processing step for transforming a source image into a new image which is fundamentally similar to the source image, pre processing results in changing the brightness of individual image pixels and segmentation is applied to subtract the foreground image from a corresponding reference background image and decompose the image into regions that correspond to objects. In second step, the shape feature extraction is performed in order to obtain the exact shape of the vehicle. It is useful in classifying and recognition of images. The third step, classifier is used for the detection of available spaces, and then finally security feature is provided to protect the vehicle from the $\mathrm{ft}$.

\section{B. Segmentation}

Segmentation is the process of separating the foreground and background regions from unwanted regions. We exclude distant regions by specifying an ROI for each camera based on the values the region occupied by the vehicle is extracted. The segmenting process is purely based on the intensity values and the edges are marked. The Region of Interest is usually determined on the basis of pixel intensity values or user determined areas. Pixels are a region that is similar according to some homogeneity criteria such as intensity and textures so as to locate and identify objects and boundaries (lines, curves, etc...) in an image. Segmentation is used to partition the image so that each pixel in the image can be identified easily. Segmentation is done to identify the edges and boundary of the objects. Segmentation is carried out in two steps; they include edge detection and thresholding. Based on the intensity at the region boundaries the edges are identified. Thresholding is the simplest technique of segmentation. Here the threshold value is computed by using some techniques such as k-means clustering or maximum entropy method based on the threshold value the image is segmented as shown in Figure 3.

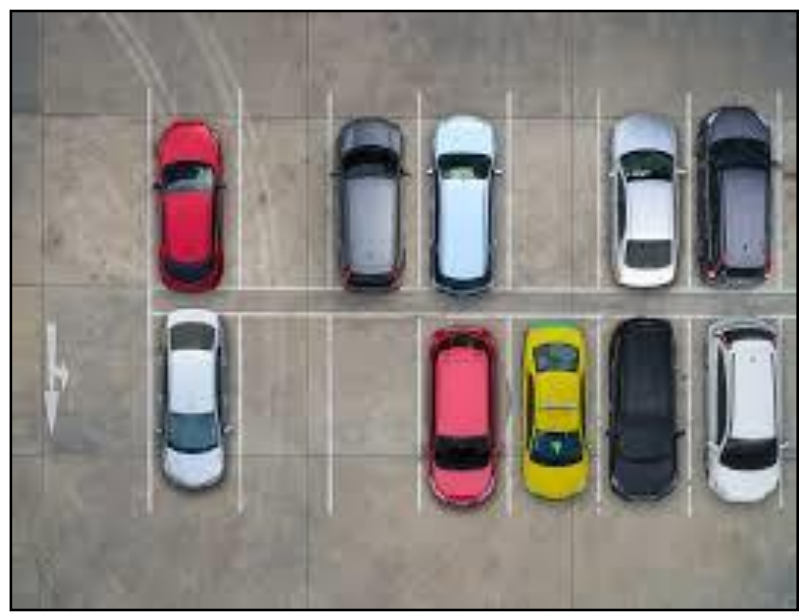

Fig 2.Input Images 




Fig 3.Segmented Image

\section{Shape Feature Extraction}

In order to reduce the amount of data, an image is represented using a set of features. Feature extraction technique is applied to get the exact specific shape of the processed image this is very useful in classifying the regions based on the attributes such as area eccentricity, centroid, convex image, orientation, extent, perimeter etc.. Among these various features only selected features are extracted that helps us to identify the shape of the vehicle as shown in Figure 4.The Grey Level Co occurrence matrix (GLCM) is one of the feature extraction techniques. GLCM is a tabulation of how often different combinations of pixel brightness values occur in an image. This gives the deviation present in the image when compared with original image by predictive image. After computing the grey level co occurrence matrix $M[i, j]$ that considers the relationship between two neighboring pixel, the first pixel is known as a reference and the second is known as a neighbor pixel then the features such as contrast, correlation and homogeneity can be computed. The contrast is defined from equation (1) represents the difference between the selected vehicle regions from the background regions that provide us to improve the accuracy of the classification of the vehicles.

Contrast $=\sum_{i, j}(i-j)^{2} M[i, j](1)$
The contrast feature alone does not help us to distinguish the vehicle types correctly hence we consider the homogeneity that is used to extract the texture descriptors of the processed image from equation (2)

$$
\text { Homogeneity }=\sum_{i, j} \frac{M[i, j]}{1+|i-j|}
$$

Vehicle types the correlation measures and energy is usually used to distinguish the vehicle types which is defined by the following equations.

$$
\begin{gathered}
\text { Energy }=\sum_{i, j} M[i, j]^{2} \\
\text { Correlation }=-\sum_{i, j} \frac{(i-\mu)(j-\mu)}{\sigma^{2}} M(i, j)
\end{gathered}
$$

Principal component analysis (PCA) reduces the information contained in a large number in original dataset into a smaller set of new composite dimensions, with no loss of information. Therefore the above method uses PCA to identify the directions of maximum variance in the 360 dimensional contour vector. The dataset with the matrix dimension $\mathrm{M} \times \mathrm{N}$ is given as input and produces an output of Eigen Vectors which is an essential coefficient and it is required as the input for the classifiers.

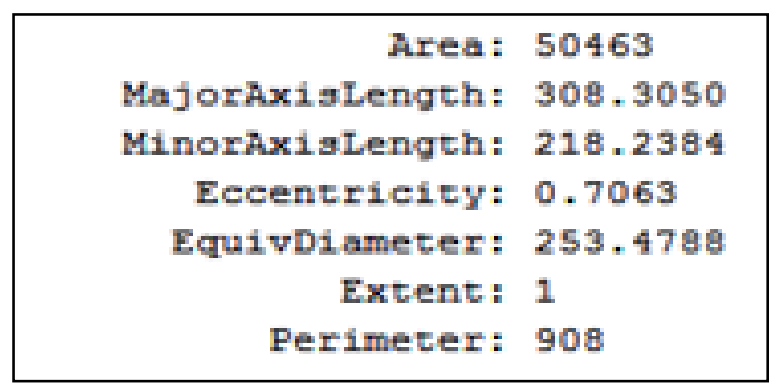

Fig 4.Attributes Extracted

D. classifier

The neural network dataset contains images taken in different time variation along with different light conditions, which includes occlusion and shadow situations also. The above condition makes the occupancy detection and the task become more difficult. (Figure:5)

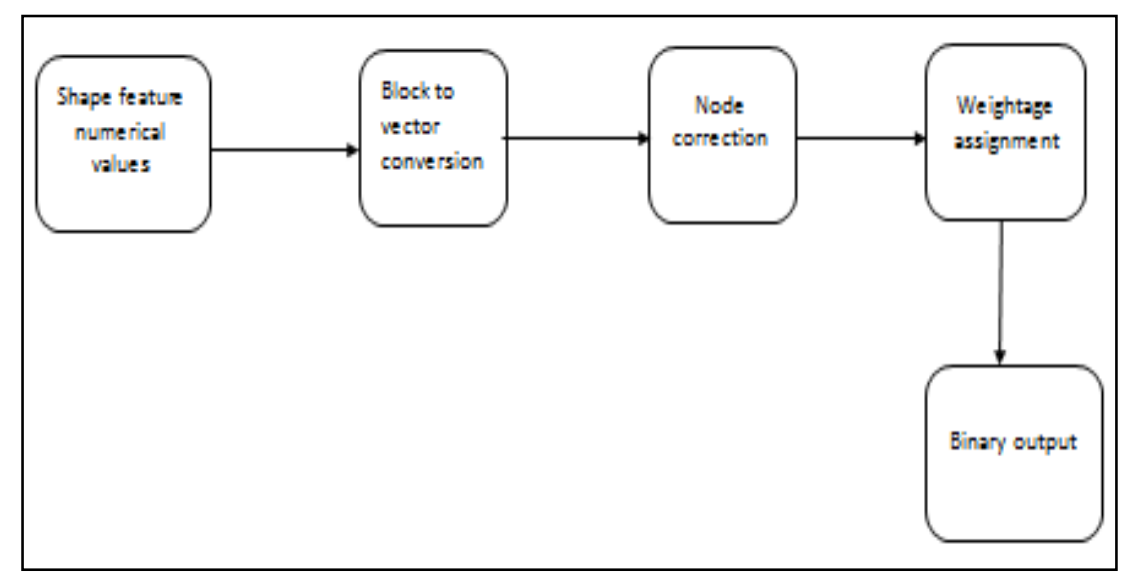

Fig 5. Block Diagram of Classifier 


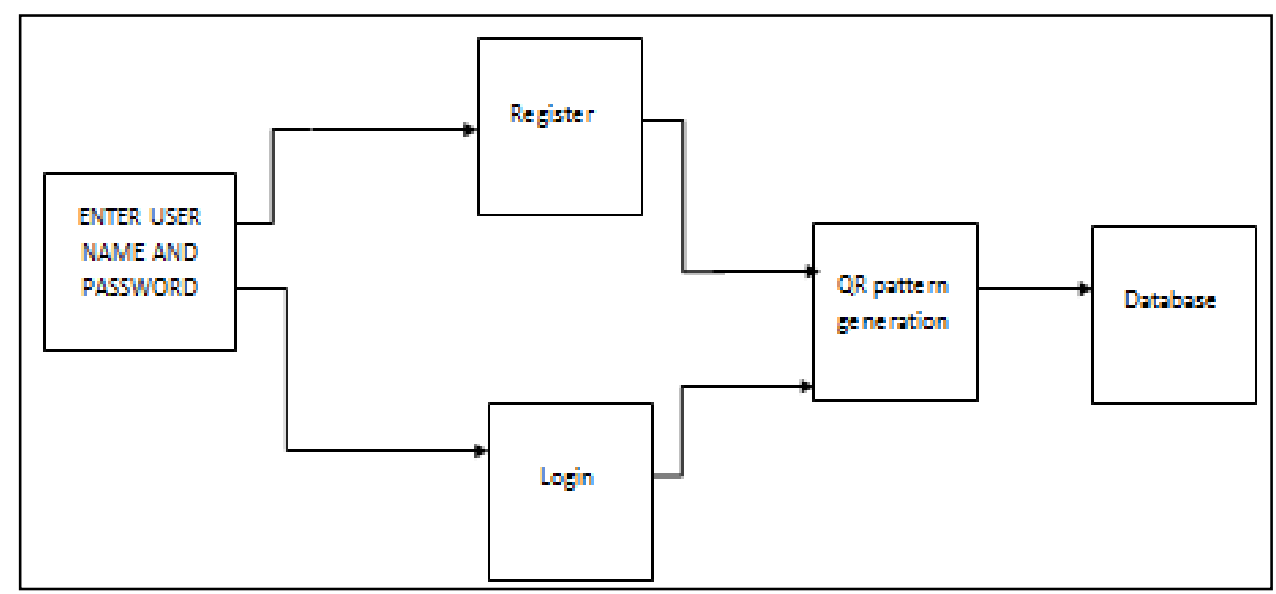

Fig 6. Block Diagram of Security

Table 1. Performance Comparison

\begin{tabular}{|c|c|}
\hline Paper Name & Accuracy \\
\hline $\begin{array}{c}\text { Identifying parking spaces \& } \\
\text { detecting occupancy using vision } \\
\text { based iot devices }\end{array}$ & $91 \%$ \\
\hline $\begin{array}{c}\text { Real time car parking system using } \\
\text { image processing }\end{array}$ & $62 \%$ \\
\hline $\begin{array}{c}\text { A cloud based smart parking system } \\
\text { based on internet } \text { of } \sim \text { things } \\
\text { technologies }\end{array}$ & $78 \%$ \\
\hline $\begin{array}{c}\text { An intelligent secure and privacy } \\
\text { preserving parking scheme through } \\
\text { vehicular communications }\end{array}$ & $51 \%$ \\
\hline Smart parking system & $93 \%$ \\
\hline
\end{tabular}

The proposed dataset has been manually and exhaustively annotated and made available to scientific community for defining new algorithms for car park occupancy detection. ANN is rarely used for predictive modeling. The Artificial Neural Networks (ANN) usually tries to over fit the training dataset. ANN is generally used in situations where the already appeared situations are collected and trained to classify the future appearance the same situations. The network consists of a topology graph of neurons, the inputs is carried on the in edges and sends the output on its out edges. The inputs and outputs are weighed by weights and shifted by bias factor specific to each neuron

\section{E. Security}

The conditional privacy for vehicle is provided using the proposed method with the help of user interface that gets the input from the user like login id and password for the particular parking slot. The registration process is performed and then if the password matches with the already registered data only then the next step is preceded. If not the access is not carried out. In this system, the usage of color $Q R$ code is used, which is an advanced form of $Q R$ code. It is an arising technology which is going to be implemented everywhere.(Figure:6)

\section{Results and Discussion}

There are several methods that are used in the detection of empty parking slot. We evaluated the performance and the accuracy provided by our system comparing to other techniques it provides an accuracy of 93\% (Table I) and an additional security by auto theft alarm system(Figure:7 9) When the vacant parking space is detected the user get notified by voice commands that direct the user to the required parking slot and also if the user wants to secure his or her vehicle then they can use the auto theft alarm system that keep track of information about the vehicle and intimate to the user whether it is secured or not .Here the performance of the proposed system is analyzed as follows. 




Fig 7. Performance analysis



Fig 8. Best training state

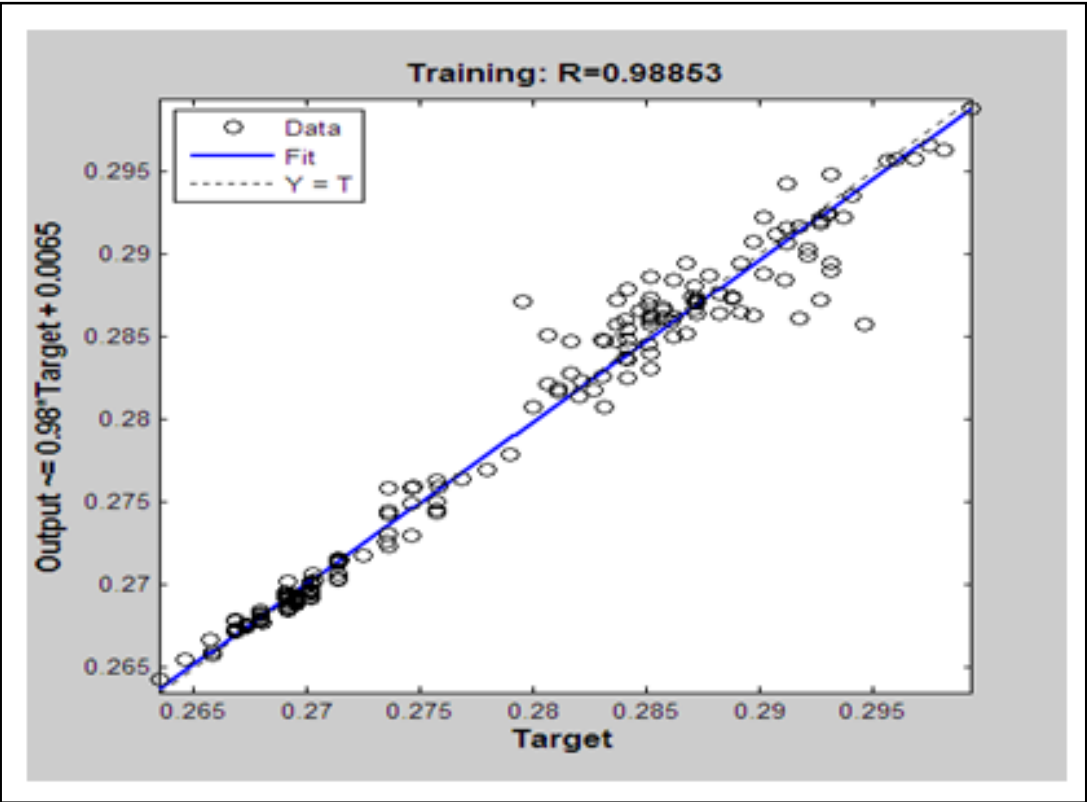

Fig 9.Similarity Match 


\section{Conclusion and Future Enhancement}

In this paper, we have presented a Smart parking system for identifying the parking slot by using classifiers and an auto theft alarm system to provide security for the vehicle. We have collected images from different angle of incidence and a trained data set is provided to the classifiers hence it is very efficient if the cameras are placed anywhere inside the parking area. If infinite numbers of training set are trained then this technique can be widely used in all parking areas. We have also tested our approach using a test set produced with a camera placed in a different place (viewpoint and perspective), with respect to the training set. The auto theft alarm system issues a login id and password for authentication purpose. This proposed idea code can be extended by using $\mathrm{QR}$ code for all government related projects throughAadhar card around the country.

\section{References}

[1] Soo mok Lee,Seung Woo Seo, Available Parking Slot Recognition based on Slot Context Analysis, IET Intelli. Trans. Sys. 10 (2016).

[2] Giuseppe Amato, Fabio Carrara, FabrizioFalchi, Claudio Gennaro and Claudio Vairo, Car Parking Occupancy Detection Using Smart Camera Networks and Deep Learning,( 2016).

[3] Junzhao Liu, Mohamed Mohandes, Mohamed Deriche, A Multi Classifier Image Based Vacant Parking Detection System,(2013) 933 936.

[4] P.Lu,X.Lin,H.Zhu, SPARK Vanet based smart parking scheme for large parking lots, (2009).

[5] Sayanti Banerjee, Pallavi Choudekar, M.K. Muju, Real Time Car Parking System Using Image Processing, (2011).

[6] K.Kaarthik, A. Sridevi, C. Vivek, Image Processing Based Intelligent Parking System, 2011.

[7] Imen Masmoudi, Ali Wali, Anis Jamoussiand Adel M. Alimi, Vision based System for Vacant Parking Lot Detection, VPLD (2013).

[8] Hilal Al-Kharusi, Ibrahim A1 Bahadly, Intelligent Parking Management System Based on Image Processing, World J. Eng. Tech., 2 (2008) 55 67.

[9] Xiao Ling, Jie Sheng, Orlando Baiocchi, Xing Liu, Matthew E.Tolentino, Identifying Parking Spaces \& Detecting Occupancy Using Vision -based IoT Devices, (2017).

\section{About The License}

(C) 2019 The Authors. This work is licensed under a Creative Commons Attribution 4.0 International License which permits unrestricted use, provided the original author and source are credited. 